\title{
Differential Ineffability and the Senses
}

\author{
STEPHEN C. LEVINSON AND ASIFA MAJID
}

\begin{abstract}
Ineffability, the degree to which percepts or concepts resist linguistic coding, is a fairly unexplored nook of cognitive science. Although philosophical preoccupations with qualia or nonconceptual content certainly touch upon the area, there has been little systematic thought and hardly any empirical work in recent years on the subject. We argue that ineffability is an important domain for the cognitive sciences. For examining differential ineffability across the senses may be able to tell us important things about how the mind works, how different modalities talk to one another, and how language does, or does not, interact with other mental faculties.
\end{abstract}

\section{Introduction}

What difference does it make to our internal mentation that we can easily talk about some domains (say, geometric figures) and not others (say, individuals' faces)? According to some, most notably Fodor, not a jot. We have the thoughts independently of the labels, in an interior language of thought, and our mental computations are entirely in that internal lingo. At the other extreme, some hold that virtually all of conscious life is embedded in language (Gauker, 1994). Most modern theorists probably stand somewhere in between, claiming that language facilitates conscious awareness, by making percepts a possible object of attention (Dennett, 1991; Jackendoff, 2007; Arbib, 2001). The literature on nonconceptual content can be read as supporting that position (Bermúdez and Cahen, 2008). A great deal of recent empirical work can also be interpreted as supporting such a view. For example, there is recent material showing effects of language on non-linguistic cognition (see, for example, Majid et al., 2004; Levinson, 2003; Regier and Kay, 2009; Wolff and Holmes, 2011). If these views are even partially right, then finding that some perceptual domains are more amenable to linguistic coding than others is to tell us something fundamental about constraints on how consciousness and reasoning can patrol our inner lives.

We thank our colleagues and collaborators on the Language of Perception Project hosted at the Max Planck Institute for Psycholinguistics, as well as Frederique de Vignemont and Gary Lupyan for comments on earlier versions of this manuscript. We would also like to acknowledge the formative influence of Moore and Carling (1988). This work also forms part of the research program 'Human olfaction at the intersection of language, culture and biology', financed by the Netherlands Organisation for Scientific Research (NWO). Parts of the manuscript were written while the second author was resident at the Swedish Collegium for Advanced Studies.

Address for correspondence: Max Planck Institute for Psycholinguistics, P.O. Box 310, Nijmegen 6500AH, The Netherlands.

Email: Stephen.Levinson@mpi.nl; Asifa.Majid@mpi.nl 
In this article, we proceed as follows. In Section 2, we unpack the notion of ineffability and outline its importance for the cognitive sciences. In Section 3 we consider to what extent there is a common vocabulary for talking about sensations across modalities. To the extent that there is a common code, this is an argument against a radical modularity within the sensorium; nevertheless, the restricted nature of this vocabulary suggests distinct limits to cross-modal coding of perceptual dimensions in language. In a sense this section is an aside, but it is an essential prologue to Section 4 where we ask to what extent there may be differential linguistic codability from one sense to another. Here we currently lack crucial data, but intuition and first principles suggest that such differential codability is highly plausible - that it is, for example, easier to talk about colors than smells. Presuming that future empirical work substantiates this, we consider how one might explain this in Section 5, suggesting two different sorts of answers grounded either in properties of language itself or properties of cognitive architecture.

\section{Is There Such a Thing as Ineffability, and If So What Is It?}

Ineffability - the difficulty or impossibility of putting certain experiences into words - is a subject that at first sight might seem to belong straightforwardly to the study of solipsism, mysticism or religion. But in fact the subject touches many empirical subjects, not least linguistics and the cognitive sciences. The subject has come up recently and perhaps most persistently under the rubric of qualia- the subjective, phenomenal aspects of experience which seem to be essential for individuating sensations and feelings. There seems to be no way for a congenitally blind man to understand what red means because he cannot undergo the experience of seeing red: red is ineffable to the blind because red is a quale, and no amount of description will make up for the actual experience (Jakab, 2001; Landau and Gleitman 1985). A quale is characterized by being a necessarily private, ineffable, conscious experience. Qualia have seemed a challenge to physicalist reductions of mental phenomena (but see Dennett, 1988). More generally, the character of first-hand experience has suggested to philosophers the need to distinguish conceptual from nonconceptual content, as for example in analogue perceptual discriminations that seem finer-grained than our concepts (see for example Gunther, 2003). Outside philosophical discussions, phenomena like synaesthesia, hallucinations, and the role of memory in interpreting experience point to the very real scientific interest of subjective experiences that resist linguistic encoding.

But regardless of qualia and what you think of them, and the broader issues of nonconceptual content, ineffability is a subject always lurking around the corner. Almost any standpoint in cognitive science makes commitments to the existence or denial of ineffabilities. Take for example Fodor's (1983, p. 125) 'epistemic boundedness' - the view that the human cognitive system puts limits on what hypotheses can or cannot be entertained. Epistemic boundedness entails that there are facts out there that are ineffable, because they are unthinkable. Another well-known doctrine is 
'linguistic boundedness' - the idea that languages are made possible only because we are endowed with a very specific language-making machine which maps grammar onto the input (Chomsky, 1986). It follows that there are conceivable languages with different properties which just don't happen to be natural human ones. The natural ones by virtue of their innate properties bound what can be said.

Quite a few eminent thinkers have denied the very existence of ineffability. The arguments usually have to do with the generativity of language and the augmentability of any lexicon (cf. Tarski, 1956, p. 164: 'Language is not something finished, closed or bounded by clear limits. It is not laid down what words can be added to this language and thus in a sense already belong to it potentially'). Thus Searle (1969) for example subscribes to the principle of expressability which holds that anything that can be thought can, in principle, be said ('of course, a given language may not be rich enough to enable speakers to say everything they mean, but there are no barriers in principle to enriching it': Searle, 1969, p. 68). Similarly, Lenneberg (1953, p. 136) states robustly 'we CAN say anything we wish in any language', it is just a question of 'the HOW of communication, and not the WHAT' (emphasis his).

Linguists have likewise subscribed politely to a doctrine of the equality of languages, implying that they all have equal expressive power, thus ruling out differential ineffability across languages. More directly, Davidson (1974) has denied the coherence of a claim that there exist mutually untranslatable languages, or that there could be conceptual schemes which are ineffable-unless we could translate them, we wouldn't be sure they were languages at all. Similarly, philosophical theories that cast conceptual content in terms of truth conditions (as in Stalnaker, 1998) suggest that any property predicated of any range of entities could as well be linguistically expressed as any other.

Against this, it is clear that a language can lack expressive power-one can for example invent a logic without a negative operator, or an arithmetical system without an additive operator: then 'there are no unicorns' or ' $2+2=4$ ' become straightforwardly ineffable. And it is patently the case that existing languages differ in expressability in specific domains. Take Pirahã, with no number or color words (Everett, 2005): in such a language, such a prosaic phrase as 'seven red tins' becomes straightforwardly ineffable. There are in fact many arguments, expertly traversed by Kukla (2005), that show that all of the demonstrations that purport to show that the concept of ineffability makes no sense in actual fact fail. Here's one simple argument in favor. Suppose, as here, we are interested in categories in language and thought. One of the things categories do is suppress differentiae in order to class tokens as members of a single class. As Nietzsche pointed out (see Kukla, 2005, p. 30), every leaf is different, so calling them 'leaves' ignores the indeterminately many ways they could be different. ${ }^{1}$ No finite set of categories, or finite vocabulary, could in principle capture all the individuating facts about this particular leaf-it

1 See Lupyan, 2008, for experimental evidence that labeling an exemplar of a chair, for example, makes you less able to remember the particular chair you saw. 
(or the conjunction of the indefinitely many facts about it) remains ineffable. Given recursive syntax, and augmentable vocabularies, this may fail as an argument against strong ineffability — as an argument against the in-principle impossibility of coding every such distinction-but it seems valid as an argument for its weaker counterpart, which insists only on the practical limitations within a language.

Before proceeding further, then, it will be useful to distinguish different kinds or degrees of ineffability (see Kukla, 2005, for a really thorough job). Weak ineffability is relativized to a specific language: sensation $\mathrm{S}$ is not codable in language $\mathrm{L}_{1}$, even though it may be in $\mathrm{L}_{2}$. Given that (as discussed above) languages are augmentable, weak ineffability must be relativized to a language at a point in time (or if one prefers, to a dialect or even idiolect at one stage of its development). This is the domain of Whorfianism, translation studies, second-language interference and so forth. Strong ineffability holds where no language at all allows the coding of sensation S (and we'll stick within the boundaries of what is epistemically entertainable, but inexpressible). Here one can distinguish different kinds of strong ineffability, quantifying over all actual, or all possible, human languages, or all languages of any kind, e.g. logical or artificial. Thus facts may be strongly ineffable in the nomological sense that in principle no language can express them, or merely in an empirical sense in that no human languages actually do so.

One further problem needs to be discussed, namely what it means for something to be expressable in a language. It will be useful to work with a strict notion of linguistic codability. We can operate with a truth-conditional criterion of codability: A state of affairs or sensation $\mathrm{S}$ is codable in language $\mathrm{L}$ if and only if there is an expression $\mathrm{E}$ of $\mathrm{L}$ such that $\mathrm{E}$ is true (or satisfiable) if and only if $\mathrm{S}$ obtains. So 'this is blue' is true if and only if the indicated thing is blue. Clearly codability in this sense is distinct from conveyability or indirect indication. For example, Yélî Dnye lacks clear color terms (or any word for 'color'), certainly none beyond white, red and black: so to convey 'It is blue' in Yélî Dnye you would have to say, for example, 'It has the surface appearance similar to the shallow sea over sand' (see Levinson, 2000). Because there is no convention for such a usage, and because this is a fresh simile, there is (a) only a degree of approximation (does the speaker have a precise blue shade in mind?), (b) a much greater latitude for interpretation, (c) much more reliance on background shared experience (how shallow a sea does the speaker have in mind?), and (d) much more room for entire misconstrual (perhaps texture rather than color is what is in the speaker's mind). Similarly, a Yélî Dnye speaker might hold up a purple fruit and say 'It looks a bit like this', with all the same unclarities of content (shape? color? reflectance?) and precision (purple or blue?). So for monolingual speakers of Yélî Dnye blue is not codable, even though it may be indirectly conveyable with some degree of uncertainty. To that extent, blue is ineffable in Yélî Dnye-weakly ineffable of course, since it can easily be expressed in languages like English.

Although truth-conditional codability doesn't rely on the prior lexicalization of concepts, in practice ad hoc descriptions tend to invite the uncertainties just illustrated. Clearly, there may or may not be a lexical item that codes for the particular attribute or object being currently conceptualized, i.e. there may be limits on lexical 
codability. To say, for example, that color is codable in English is to say that there are words which code only the descriptive, abstract property of color and not other properties such as reflectance, texture, shape etc. The specific qualities of smells, on the other hand, are not lexically codable in English since there are arguably no words that identify the precise properties of smells. Words such as stinky and fragrant code for affect, not odor attributes, while 'smells like a rose' identifies an object as typical source, not an odor quality. Thus, your perfume may seem stinky to us, but fragrant to you (in contrast your blue will not be our red). The issue of lexical codability becomes more complex when we think not just of the attribute, property or object being conveyed, but the range of exemplars which are being encapsulated with a term (à la Nietzsche).

When a source-object or exemplar is specified, as in lilac odor, an additional distinction between opaque and transparent sensory descriptions becomes clear: lilac odor is transparent when it refers to odors caused by lilacs whatever the experience is like, and is opaque when it refers to the subjective experience, whether or not it is caused by lilacs (Clark, 1993, pp. 1-3). There are thus two possible worlds being described: the objective world where things have properties, and the subjective world of qualia themselves. Without any special language for a domain, we can still explore the dimensionality of the subjective world by doing multidimensional scaling over similarity judgments of things in the objective world (Clark, 1993) — words bring categories into this subjective world, by bundling those judgments. When a language has words for classes of percepts they will often be ambiguous (or more likely semantically general) over transparent or opaque readings: red may be predicating objective properties (the box is red) or subjective judgments (in this light it looks red). Domains themselves may differ here: quarrels about color lend themselves to objective resolution in a way that disagreements about taste or smell do not. In addition, there is fine-grained lexicography here yet to be done. When words denote sources (like lilac), and only indirectly sensations, they are more likely to have transparent readings; where there are special abstract terms directly for sensations (e.g. red) they may primarily have opaque readings. No one seems to have done either the conceptual or lexicographical work required to explore this distinction.

The notion of codability was extensively explored in the 1950s and 1960s. Brown and Lenneberg (1954) constructed a multifactor notion of codability based on (a) the number of syllables in a description, (b) the number of words, (c) reaction times in naming, (d) consensus across subjects, (e) consensus within subjects across time (these measures correlated well, but (d) has the largest factor loading by far). As later formulated, 'codability is a measure of the efficiency with which either a color or another sensory experience may be transmitted in a given language code' (Lenneberg and Roberts, 1956, p. 20; see Lucy, 1992, Chapter 5, for discussion of this tradition.) This hybrid psycholinguistic measure, which we can call efficient codability - has had continuing influence in, for example, the work on color terms, so that Berlin and Kay (1969) defined a basic color term as a monolexemic superordinate term with psychological saliency (measured, for example, by recall lists and consensus). Subsequently another measure called communication accuracy was developed by Lantz and 
Stefflre (1964), using describer-matcher tasks where participants had to pick color exemplars out of an array on the basis of a description; recent replications suggest that this shows an advantage for focal colors (colors which are judged to be best exemplars), although only in ordered arrays (Agrillo and Roberson, 2009). Perhaps the difference between efficient codability and communication accuracy is best thought of as a difference between how easily an object or attribute can be encoded versus decoded.

To sum up, there are three notions of codability that we need to distinguish: linguistic codability (contrasting with indirect conveyability), efficient codability (a psycholinguistic measure), and communication accuracy. The first, linguistic codability, is in principle discrete (yes or no), while the other notions are gradable. Some state of affairs or sensation $\mathrm{S}$ will be (weakly) relatively ineffable to the extent that it resists codability in language $\mathrm{L}$ by any of the three measures (i.e. coding in $\mathrm{L}$ is linguistically impossible, inefficient or inaccurate or a combination thereof), compared either to some other domain in the same language, or the same domain in another language. For example, we might want to say that smells are relatively ineffable compared to colors in English. We might also want to say that colors are relatively ineffable in Yélî Dnye (which lacks developed color terminology) compared to English.

\section{Comparing Percepts Across Sensory Modalities}

Since we are interested in variable ineffability across the senses, we need to consider intrinsic similarities and differences between the modalities. There is a long tradition of study in this area (see for example Marks, 1978; Spence, 2011), and indeed the story begins with Aristotle, who in De Anima posited the existence of common sensible attributes (e.g. magnitude, shape, roughness, sharpness can be both seen and felt). Galileo posited four such primary qualities (size, shape, quantity and motion), to which Locke added solidity, while Kant held that space, time, number and quantity are suprasensory qualities (see Marks, 1978, pp. 12-13). According to philosophers, primary qualities, such as number, are independent of specific sensory modalities - you can see them, feel them, hear them; secondary qualities, however, can only be appreciated by a single sense-you can only experience hue through the eyes, taste through the tongue, and so forth. Wundt took up some of these ideas, starting an experimental tradition comparing, for example, the perception of physical length. It turns out that for visually perceived length and length felt by a moving arm, perceived length closely matches actual length, while visual length and length felt by grip between fingers (proprioceptive length) do not match (Marks, 1978, pp. 15-20). Shapes can be cross-modally recognized by sight and touch (although the haptic sense is notably inferior): even the Müller-Lyer illusion works in both modalities (Gentaz and Hatwell, 2004). Duration is another quality independent of modality, or at least perceivable through different modalities. Perceived durations of events are proportional to actual durations whether perceived through vision or 
audition, but visual events seem shorter and may even be less precise (Zélanti and Droit-Volet, 2012).

In addition to primary or suprasensory qualities - qualities that transcend a single sense - there are also analogous dimensions across the senses. Amongst the candidate cross-modal dimensions of this kind are size, intensity, and brightness (see Marks, 1978, pp. 52ff). More recently, Walsh (2003) has proposed a single magnitude estimation system, apprehended across senses, that works across space, time and number. As a primary quality size can be estimated both visually and tactually, as mentioned; but as an analogous dimension sounds can also be felt to have large or small extent independent of pitch (consider the sound of an organ versus that of a violin). Experimentally, it can be shown that the perceived size of vibratory stimuli varies inversely with the frequency of the wave. To some extent the perceived size of sounds matches the size of the generator, an effect used much in animal displays (see for example Fitch and Reby, 2001).

Intensity is another candidate cross-modal dimension. Lights, sounds, smells, tactile pressures, tastes, pains, emotions can all have low or high intensities. Subjects can systematically match intensities across modalities. Weber's Law (1834) states that intensity increases reach levels of discrimination according to a fixed proportion: if a sensation of unit 1 can just be differentiated from one of unit 10, then 10 can just be discriminated from a 100, and 100 from a stimulus of 1000, etc. Modern psychophysics roughly corroborates this across odor, taste, touch, temperature, loudness and light perception (Laming, 1989). Similarly, magnitude estimations, regardless of modality, follow Stevens' Law (1957), namely that perceived intensity is proportional to physical intensity raised to some power, or equivalently 'equal stimulus ratios produce equal sensation ratios' (Marks, 1978, p. 132) (e.g. 2:1 ratio of loudness $=8: 1$ ratio of acoustic energy).

Finally, consider brightness: smooth, hard, sharp, light, cold things and events are bright, while the counterparts (rough, soft, blunt, heavy, warm) are dull (von Hornborstel, 1927). Brightness may equate to intensity in the case of light, but in the case of sound it involves both relative high pitch and amplitude, as can be shown by cross-modal matching experiments (e.g. Marks, 1989).

Another way to see parallels across the modalities is to consider each modality as consisting of a space with opposing poles on a number of dimensions. As Aristotle pointed out 'All sensible objects contain the principle of opposition' (De Anima, quoted in Marks, 1978, p. 63). Through a long history of psychophysics, the perceived color space has come to be thought of as a distorted sphere, with two dimensions of opposition (brightness, saturation) and one circular dimension of hue. Henning (1916) posited that taste space is a tetrahedron (3-sided pyramid), with opposed vertices sour-bitter-salty-sweet, and multidimensional scaling of taste judgments is thought to support this structure (Clark, 1993, p. 142). Henning also proposed an odor perceptual space, in the shape of a prism with six vertices (Clark, 1993, pp. 143-6), whereas some modern theories suggest a single dominant dimension, i.e. pleasant versus unpleasant (Khan et al., 2007; Yeshurun and Sobel, 2010). Nevertheless, odor space continues to elude satisfactory 
characterization (Murphy, 2011). Auditory space may seem just two dimensional, with tone and loudness, but for each tone in a chord we must add another pair of values on these dimensions. (All these senses also have a spatial localization, adding an additional three-dimensions - note for example that the location of a color patch in the visual periphery renders it grey.) Clark (1993) gives a spirited defense of multidimensional scaling as an objective technique for mapping subjective sensory spaces, and shows how the interpretation of these spaces can sometimes be correlated with neurophysiological dimensions.

Synaesthesia suggests that cross-modal matching is a natural tendencysynaesthesia may only be experienced by around 5\% of adults (Simner et al., 2006), but some scholars have estimated that up to $50 \%$ of children experience the condition (see references in Marks, 1978, p. 102). A recent study, the first to use randomly sampled children, has actually found a modest prevalence of only $1.3 \%$ grapheme-color synaesthetes amongst 6-7-year-olds, in line with the $2 \%$ prevalence for the same condition reported in the adult population (Simner et al., 2009). Simner et al.'s study suggests a complex developmental sequence to the condition which requires further study.

There was a tremendous interest in synaesthesia in the 1930s, then almost none until the recent growth of neuroimaging techniques that make direct inspection of activity in different sensory areas possible. Some of this work reinforces the idea of analogous cross-modal dimensions - for example light colors are overwhelmingly associated with high notes (Ward et al., 2006), and vowel sounds have systematic color associates (Marks (1978, p. 88) tabulates 70 years of studies; see also Simner, 2007). Obviously, these results tie into sound-symbolic associations amongst normals (non-synaesthetes).

The ability to match sensations across modalities is of course also rooted in experience, because events are naturally multimodal (a point made by Bishop Berkeley in 1709). If you are standing at a station, and a train is approaching, as the train fills more and more of the visual field, the sound also increases, until one actually feels the increasing rush of air; as it departs, the whole process of linked sensations is reversed. The processes by which cross-modal mappings are formed and maintained are still little understood, however. Some scholars would have it that cross-modal mappings are an inevitable consequence of exuberant synaptic connections during brain development (Maurer et al., 2012), but others have highlighted the role of experience (Howes, 2006; Lewkowicz, 2011). Cross-cultural evidence could help shed some light on the matter. A recent study by Bremner and colleagues (2013) suggests that some associations (e.g. sound-visual shape) may be relatively stable across cultures, while others (e.g. taste-visual shape) are more variable.

Finally, one should consider the role of language in cross-modal connections. A number of authors (e.g. Dennett, 1991; Spelke and Tsivkin, 2001; Mithen, 1996) have suggested that the special role of language in human cognition is to provide a new channel for cross-modal communication - language bridges the specialized cognitive modules acquired over eons of vertebrate evolution as independent, non-cooperating specialist faculties. In the case of spatial frames of reference, there 
is an argument that language plays a role in regimenting a preferred frame of reference across modalities, so that gesture, language, spatial memory and navigation strategy can be directly coordinated (Levinson, 2003; Haun et al., 2006). Likewise, where languages vary in their cross-modal metaphors, speakers correspondingly vary in their propensity to make cross-modal mappings outside of language (Dolschied et al., 2013).

Whether language sets up cross-modal correspondences, or only reflects them, nevertheless it is obvious that linguistic labels name vertices on cross-modal dimensions like intensity: we talk of bright/dull sounds and lights, sharp/faint pains and sounds, big noises and objects, long/short things and durations, loud/soft sounds and colors, intense sounds, pains, colors. We also talk about pure tones and hues, hard surfaces and pains, revolting odors and colors, sweet tastes and tones. And the shared opponent, gradient character of the dimensions of sensory spaces is reflected in the use of shared intensifiers (very/truly/intensely red or sweet or loud), while the language of hedges may indicate a shared use of prototypes across the senses (c.f. sort of red or sweet or loud).

\section{Differential Ineffability Across The Senses}

Introspection suggests that different modalities, and submodalities, are differentially codable. It is arguable for example that, in English at least, it seems generally easier to linguistically code colors than (non-musical) sounds, sounds than tastes, tastes than smells. Within the visual domain, object shapes seem easier to verbalize than faces (it is easier to describe the shape of a vase than your grandmother's face), while within the tactile modality textures seem easier than pain. Clearly, there are also expertise effects - a sound-effects expert may be better on sounds than on colors, and a perfumer better on smells than textures; specialist walks of life provide both experience in discrimination and the vocabulary to go with it. Nevertheless, English thesaurus lists show many more column inches for color than sound, sound than taste, taste than smell, suggesting that the ordinary vocabulary is much denser for color than the other modalities.

The potential systematicity of this may be buttressed by a number of observations. First, it is usually assumed that the senses are not equal. One might establish this by counting voxels of dedicated grey matter (see, e.g., Golland et al., 2007), but it can be simply illustrated with mental imagery. While people report no difficulty in conjuring a mental image of their bedroom or a giant, green spider (close your eyes - imagine it), many people find it very difficult, if not impossible, to conjure a specific smell, such as banana or rose (can you imagine the odors?). Tactile texture may be somewhere in between. Try to imagine the feeling on your tongue if you were to lick a carpet. This is something most readers may have not bothered to experience, and yet the feeling can be quite easily entertained (see Brower, 1947).

In direct perception the senses are not equal either. For example, if vision and audition suggest a slightly different spatial source of a perceptual event, vision always 
wins (hence the possibility of ventriloquism). The distance between two points is judged by touch to be much smaller than it actually is, while visual distance judgments remain veridical (Stevens, 1975; Gescheider, 1997). Together with the relative amount of cortex dedicated to vision compared to other senses, this makes clear that vision is the dominant human sense. So we might expect language to be more attuned to visual discriminations. Note, however, that within a domain, such as vision, there are subdomains that seem strongly ineffable, like describing what someone's face looks like (i.e. identifying a person by facial description), a matter discussed below.

There may be other ways to arrive at such conclusions. Consider the philosophical literature on nonconceptual content, where in contrast to conceptual content, direct perceptual content is thought about as analogue, non-metric, hugely fine-grained and context-specific (Bermúdez and Cahen, 2008). Could it not be that some perceptual domains are more like that than others-for example, more fine-grained because of the psychophysical properties of the domain? Here one might try to rank the senses in terms, for example, of their psychophysical multidimensionality: Color may have three such dimensions, taste four or more, and so forth. Then there is the question of the number of just-noticeable-differences - the jnds - within these spaces. The size of the spaces our senses traverse would then have a direct bearing on their susceptibility to categorization.

Although these arguments are quite persuasive, they rely on common sense observations among English speakers. It may be that from the point of view of other languages and their speakers, the pecking order across the senses might seem rather different. In other words, is this relative codability of the different senses a matter of strong ineffability (across all languages) or only weak relative (i.e. language-specific) ineffability? To decide this, we clearly need data from many languages other than English. A problem here will be to find ways of equally sampling the different psychophysical spaces, to give each domain a chance to shine in each of the languages explored. A study of this kind is under way by the present authors and colleagues, where we examine across more than 20 languages (including 3 sign languages) whether the senses are of equal efficient codability (à la Brown and Lenneberg). But it will be some time before we have systematic empirical evidence to report (although see Majid and Levinson, 2011, for an interim report).

Meanwhile, it is already interesting to explore cross-cultural variability in the categorization of a single domain, because that gives us a sense of the variability in coding we may expect on a cultural basis. Here color terminology is the best explored domain (see the World Color Survey http://www.icsi.berkeley.edu/wcs/ for example), and shows extensive variation in the density of coding of the color space across languages (from just two terms to fifteen 'basic' terms (Roberson et al., 2008), and up to thousands of ancillary terms). Taste terminology seems more restrained, but even here the degree of distinct labeling of the sweet, salt, sour, bitter apices of the taste space varies considerably-some languages collapse sour + bitter, others sweet + salt, yet others sweet + salt + sour or sour + bitter + salt (Majid and Levinson, 2008). And odor terminology turns out to vary from just one 
abstract term ('smell') to a dozen or more primary terms that directly label classes of odorants (see below). What these facts show is that there is enough variance across languages to make the question of whether there are stable preferences for differential coding across the senses anything but a closed issue.

\section{Explanations of Ineffability}

If we find differential ineffability across the senses, what might be its roots? Obviously, it will be important to know if such ineffability is strong (across all languages) or weak (in just some), and how relative (e.g. to other senses) or absolute it is. In principle, weak ineffability (say ineffability in most languages) might be rooted in principles of strong ineffability, which some language-users have struggled to partially overcome. So let us start by considering explanations for strong ineffability. Such explanations would seem to be of two types, which we will discuss under the rubrics cognitive-architectural and limits of language. Cognitive-architectural explanations argue that language intrinsically has poorer access to some cognitive modules than others; while limits of language explanations locate the bottleneck in design properties of language itself.

\subsection{Cognitive-architectural Explanations}

Let us start with the first kind of explanation which focuses on cognitive architecture. Consider face-description for recognition. We have all struggled to describe the person who is to be met at the station by someone else (Moore and Carling, 1988; see Ellis et al., 1980, for the kinds of descriptions people do give for faces). It is unlikely that English alone is peculiarly deficient here. If it were easy to remedy, there would be expert lingo for police identification, or beauty parlors, model agencies, art history, or the like (see http://www.face-rec.org/research-groups/for the large amount of research being conducted into machine recognition of faces). So there are reasons to think that facial recognition features are strongly ineffable. Why? One natural kind of explanation would be focused on the internal communication between mental modules and faculties, and such an explanation might suggest that face-recognition is an ancient mammalian trait (sheep do it too), associated with a specific brain area (the fusiform gyrus) - so it is a classic Fodorean encapsulated module, that takes visual input and can only deliver its results to our (non-encapsulated) mental list of significant others. We can name them, but not describe them. This would be an explanation in terms of cognitive architecture. It predicts that we would be equally bad at, say, drawing a person's face from memory, however good we were with a pencil.

Instead of representations being encapsulated, it could be that the cortical resources required to process language and a certain sensory modality interfere with one another, as has been claimed for olfaction. According to Lorig (1999), the reason people find it difficult to name odors is because both language and odors 
are processed using similar neural networks because, according to him, they both share complex temporal signatures. Here the problem is not encapsulation but competition for resources.

Another architectural kind of explanation might focus on the content of the representations themselves. The content might be multiplex, for example, as seems to be the case for pain (Perl, 2007), and thus not easily graspable (Bonch-Osmolovskaya et al., 2009). For face-identification it could be argued that the system is dealing with a three-dimensional object that is peculiarly deformable, so that isolating out the underlying facial structure from the transient expressions is a complex process-language may have more access to the superficial expressions than the inferred underlying structure (so we would have more words for facial expressions like smile, frown, wink, furrow the brow, than we would have for different types of facial structure) - and, in fact, these dynamic aspects of face recognition are subserved by different functional and neural systems (Haxby et al., 2000; O'Toole et al., 2002).

Yet another explanation would be in terms of memory. It just so happens, the story would go, that some sensations yield very reliable recognition memories, but the same sensations can never be actively recalled without the presence of the stimulus. Good candidates, apart from faces, would be odors, and the shared property might be the sheer complexity of micro-discriminations of multiple features in the stimulus. According to the latest version of the American Chemical Society database, there are around 5.8 million potential odorants - i.e. chemicals with a molecular weight below 300 Dalton. Humans are capable of discriminating potentially an infinite number of odors, which can be constructed from complex mixtures of these odorants (Matthias Laska, personal communication).

Finally, only aspects of sensation accessible to consciousness will be accessible to language, because language use is itself a deliberative sequence of actions. There are many aspects of mental life that are not accessible to consciousness, perhaps because there was no evolutionary advantage to deliberate over them, and even deleterious effects to such access. Face identification might be such a process - we can act on the identity, but not normally on the very process of finding the identity. ${ }^{2}$ Similarly, it could be argued that olfaction is critical for subconsciously motivated behaviors (Stoddart, 1990), making it less amenable to consciousness and language.

The relative accessibility of different sensory modalities to consciousness and language may reflect the ancient course of phylogeny. On the one hand, early-acquired senses may be more encapsulated since they originally existed without their peers. On the other hand, some of our senses may have atrophied at the expense of the

\footnotetext{
2 The same holds for discrimination of faces based on gender. Infants as young as five months can discriminate male and female faces (Fagan and Singer, 1979) and it takes adults only $600 \mathrm{msec}$ to classify faces according to their gender (Bruce and Green, 1987) with an extremely high accuracy rate $\sim 96 \%$ (Bruce and Humphreys, 1994). Yet, we do not have a good understanding of the features involved in this process. Neither first-order features (e.g. mouth width; eye-to-eyebrow distance), nor second-order features (e.g. the angle from point of chin to the mandible at mouth line) suffice to deliver classification as good as humans can achieve (Bruce and Humphreys, 1994).
} 
increasing dominance of others. Comparison of human and ape genomes show accelerated evolution in some human hearing and olfaction genes (Clark et al., 2003), but overall attrition of olfaction in favor of color vision (Gilad et al., 2004). Language as a late-comer may have enhanced access only to those senses under selection during its own evolution.

\subsection{Limits of Language Explanations}

In contrast to cognitive architecture explanations, the limits of language argument would suggest that there is something specific about the nature of language coding that makes some sensations easier or more difficult to express. The information is accessible, but just not codable. Even if there were a good vocabulary for kinds of eye-shape, nose-shape, mouth-shape, etc., description of faces would be difficult, according to this account. Anyone who has tried to draw a portrait knows that it is the precise placement of such features with respect to one another that seems to yield an identifiable face. No language seems able to provide this kind of precise description of spatial ensembles. One factor may be that spatial language is attuned to the relation of two, maximally three, entities (Levinson, 2003; see also Landau and Jackendoff, 1993). Another factor may be the generally poor metric properties of language, even in proportional information: we don't expect to find a lexical item meaning 'a mouth that is wider than the distance between the two pupils', or 'a nose that projects for a third of its length beyond the plane of the forehead', let alone 'a nose that makes an angle of 135 degrees with the forehead'. In any case, whatever the reasons, describing faces such that recognition is possible from the description alone seems beyond the power of language, even when within the power of conscious observation.

Arguments about the limits of language are likely to focus on the general intrinsic limitations imposed by the design features of a natural language, to do with the nature of the linguistic code, size of lexicons, features of semantics and syntax, or learnability. Let's consider each of these in turn.

Language is 'digital', compared for example to the analogue nature of gesture. This fact, combined with finite lexicons (discussed below), ensures limits to the granularity of linguistic discriminations: one can easily gesture a precise contour shape, while one's language may or may not have the relevant lexicon (e.g. ogee). Sign languages present an interesting case here-the medium of the language may lend itself to more precision for some aspects of conceptual content than spoken languages. Because signs are produced in three-dimensional space, they can iconically represent shapes, locations, and movements in a way that spoken languages cannot (Taub, 2001). Despite the affordances of the modality, however, signers still interpret forms in a 'digital', i.e. categorical fashion, even though they can manipulate them to be analogue (Emmorey, 2002).

It is unclear to what extent language modality really matters for ineffability. For example color lexicons in sign languages parallel those in spoken languages (Woodward, 1989). Likewise, although there are distinctive hand movements to explore 
different tactile qualities — such as texture, weight, and temperature - and therefore these movements could be used to form object signs, the sensory qualities do not seem to be sufficiently salient to do so. Instead shape and size are exploited, just as they are in spoken languages (Goldin-Meadow, 2007).

A language's lexicon is finite, and working vocabularies are relatively small (say of the maximum order of 50,000 producible items), and although the generativity of language can give us 'workarounds' (of the kind found in a dictionary, where for example 'ogee $=$ double continuous curve, with reversing convexity'), the finite stock still gives a fundamental granularity which is hard to break through without evolving new lexical items with their associated conventions, which in turn need to be accepted by a community.

Finite vocabularies presuppose categories - that is to say, a word (leaving aside demonstratives and proper nouns) generally describes a type (or property). Percepts that come only or largely in tokens may resist coding-thus although there may be gross types of faces (according to gender, age, race, etc.) or odors (pleasant, unpleasant, strong, weak, etc.), the identity of a face (and possibly odor) is effectively a singleton, in contrast to the way a color or tone can be a direct and near perfect instantiation of a type.

Cross-linguistically universal design features of semantics and syntax may restrict codability of some dimensions. Consider for example predicate argument structure: natural language predicates are maximally ternary, it seems, ${ }^{3}$ thus making it difficult to encode large ensembles, as mentioned above. Then there is the lack of metric precision in lexical meaning mentioned above, which may ultimately derive just from constraints on the size of the lexicon.

Learnability may also impose severe restrictions on the size of lexica and the complexity of syntax. Consider just this mundane fact: to learn the meaning of a referential word we must have access to the referent, or at least be able to triangulate the nature of the referent from other words and facts. Further, for a community to share the meaning of a word, they must all have direct or indirect access to the referent. But qualia by definition do not meet these conditions, and such subjective matters as qualities of pain or emotion would seem to resist objective conditions for word use - the best we can do is use such words with a reference that matches how we would feel or act in similar circumstances. ${ }^{4}$

Understanding further these intrinsic limits of language is one of the central goals of the study of ineffability. Depending on one's stand on, for example, the relation of language to consciousness, or language to reasoning, these limitations may constitute fundamental constraints on other aspects of human cognition.

3 More arguments can be optionally incorporated in a statement by adjuncts or conjuncts of course, as in 'John gave Mary the book for Sam and Sue', but the inbuilt design constraint is three subcategorized arguments per predicate.

${ }^{4}$ See Levine's (2005) attempt at elucidating what people mean when they talk of 'love' based on his years of experience as a clinician. 
So far we have considered only the kinds of explanations that might be adduced for strong ineffability. As mentioned, these same factors may be universally applicable, but only make for weak ineffability (i.e. ineffability in some, perhaps in most languages) if they are gradient in nature. For example, odor perception may tend to be ineffable in most languages, but expert interest (as with perfumers or odor researchers; see Agapakis and Tolaas, 2012) or cultural preoccupation might overcome this (as with the Jahai, a group of nomadic hunter-gatherers of the Malay Peninsula; see Burenhult and Majid, 2011). Factors involved in strong ineffability could then bias the statistical outcome that we recognize as weak ineffability.

But weak ineffability could have intrinsic cultural roots of its own. It is arguable that many of the developed vocabularies of English and other European languages for the phenomenal world are the product of long and involved histories - for example, our color terminologies seem to owe a great deal to the antique and medieval cloth trade (Gage, 1995). Consider for example that the Vatican has maintained a reference set of 30,000 labeled color chips since the 1500s, in order to reproduce mosaics. Cultures without applied color technology show very limited abstract color terminology, if any (Levinson, 2000). To that extent, underdeveloped coding of sensory domains may simply reflect lack of cultural preoccupation. People without musical instruments (like the Rossel Islanders) may have little use for a metalanguage for tone, cultures with limited cuisine or puritanical attitudes to self-embellishment may not be conducive to elaborate vocabularies of taste or smell, and so forth. In the other direction, there are persistent ethnographic reports of languages with more elaborate basic lexica for smell (e.g. in Totonac: Aschmann, 1946; or Jahai: Burenhult and Majid, 2011) than is found in English. In general, though, it seems quite plausible that the process of manufacturing objects of recurrent type would require verbal means for talking about the abstract properties of things on individual dimensions - the color, texture, transparency, weight and dimensional qualities that are the sort of things checked by consumers or quality control. In contrast, in many societies with simple technologies, things come packaged by nature with their cross-modal properties (a ripe mango has a certain color, taste, texture, shape, etc.), and there would be little point in isolating out the different dimensions. Take for example the exuberant Nilotic cattle color terms, which code pattern, i.e. shape distribution as well as the color of cattle skins (of the kind: speckled black and white with black head: Evans-Prichard, 1934). Such pre-industrial lexical elaborations may trade off relative ineffabilities in single sensory fields, with high codabilities of recurrent cross-modal types of cultural importance.

\subsection{The Implications for Future Research}

The arguments of this article have the following implications for future research. As noted earlier, it is simply unknown whether the difficulty to describe individuals' faces, odors or emotions are the result of strong or weak ineffability, and whether such ineffability is due to cognitive architectural constraints or due to the limits of language. So we first need to establish the basic facts. We have distinguished three 
different kinds of codability that could be studied: lexical codability (i.e. the resources in the language), efficient codability (i.e. a hybrid psycholinguistic measure) and communication accuracy (i.e. how easy is it to decode the message). As mentioned earlier, we have begun to examine efficient codability across diverse languages but many things remain to be tested. For example, across diverse languages how similar or different is lexical elaboration across sensory modalities? Are there always more words for visual phenomenon, followed next by auditory, then tactile, taste and smell qualities? More generally, is there a single hierarchy of lexical codability across languages? If not, then what factors drive linguistic communities to elaborate in some domains rather than others? Corpus studies or free-listing methods could untangle some of the issues relating to lexical codability.

Another issue that could fruitfully be explored is communication accuracy, and its relationship to the other notions of codability. Does it make a difference to communication accuracy if a notion is lexically codable or not? Perhaps we can do an equally good job at expressing ideas through indirect conveyance (cf. Lucy and Shweder, 1979). Describer-matcher tasks across different sensory modalities could tease these factors apart. It will take a different set of studies again to be able to disentangle the various explanatory factors we set out above. We hope this article serves to prompt researchers to venture into this important but underexplored area.

\section{Conclusions}

The notion of ineffability has an unmistakable metaphysical whiff about it, but we hope to have made a case that, first, the relevant notions can be more or less precisely defined, second that ineffability seems to vary across sensory domains, and third that interesting rival explanations might be marshaled to account for the differences, each of which suggests specific lines of empirical research. To decide between these different lines of explanation we will need a lot more data, but the indications are that there is every reason to think that the empirical study of relative ineffability, across modalities and across languages, may yield fundamental insights into mental architecture, the intrinsic limits of consciousness and language, and the degree to which cultural preoccupations can (or cannot) override or adjust to these constraints.

Language and Cognition Department Max Planck Institute for Psycholinguistics; Radboud University Nijmegen

Center for Language Studies Radboud University Nijmegen

\section{References}

Agapakis, C. M. and Tolaas, S. 2012: Smelling in multiple dimensions. Current Opinion in Chemical Biology, 16, 569-75. 
Agrillo, C, and Roberson, D. 2009: Colour language and colour cognition: Brown and Lenneberg revisited. Visual Cognition, 17, 412-30.

Arbib, M. 2001: Coevolution of human consciousness and language. Annals of the New York Academy of Sciences, 929, 195-220.

Aschmann, H. P. 1946: Totonac categories of smell. Tlalocan, 2, 187-89.

Berkeley, G. 1709: Essay Towards a New Theory of Vision. Dublin: Jeremy Pepat.

Berlin, B. and Kay, P. 1969: Basic Color Terms: Their Universality and Evolution. Berkeley, CA: University of California Press.

Bermúdez, J. and Cahen, A. 2008: Nonconceptual mental content. In E. Zalta (ed.), Stanford Encylopedia of Philosophy, http://plato.stanford.edu/entries/contentnonconceptual/

Bonch-Osmolovskaya, A., Rakhilina, E. and Reznikova, T. 2009: Conceptualization of pain: a database for lexical typology. In P. Bosch, D. Gabelaia, and J. Land (eds), Logic, Language and Computation. Berlin Heidelberg: Springer-Verlag.

Bremner, A. J., Caparos, S., Davidoff, J., de Fockert, J., Linnell, K. J. and Spence, C. 2013: 'Bouba' and 'Kiki' in Namibia? A remote culture make similar shape-sound matches, but different shape-taste matches to Westerners. Cognition, 126, 165-72.

Brower, D. 1947: The experimental study of imagery: II. The relative predominance of various imagery modalities. Journal of General Psychology, 37, 199-200.

Brown, R. W. and Lenneberg, E. H. 1954: A study in language and cognition. Journal of Abnormal and Social Psychology, 49, 454-62.

Bruce, V. and Green, P. R. (eds) 1987: Visual Perception. Hove: Erlbaum.

Bruce, V. and Humphreys, G. (eds) 1994: Object and Face Recognition. Hove: Erlbaum.

Burenhult, N. and Majid, A. 2011: Olfaction in Aslian ideology and language. The Senses \& Society, 6, 19-29.

Chomsky, N. 1986: Knowledge of Language: Its Nature, Origins and Use. New York: Praeger.

Clark, A. 1993: Sensory Qualities. Oxford: Oxford University Press.

Clark, A. G. et al. 2003: Inferring nonneutral evolution from human-chimp-mouse orthologous gene trios. Science, 302, 1960-3.

Davidson, D. 1974: On the very idea of a conceptual scheme. Proceedings and Addresses of the American Philosophical Association, 47, 5-20.

Dennett, D. 1988: Quining qualia. In A. J. Marcel and E. Bisiach (eds), Consciousness in Contemporary Science. Oxford: Oxford University Press, 42-77.

Dennett, D. 1991: Consciousness Explained. New York: Penguin.

Dolscheid, S., Shayan, S., Majid, A. and Casasanto, D. 2013: The thickness of musical pitch: psychophysical evidence for linguistic relativity. Psychological Science, 24, $613-21$. 
Ellis, H. D., Shepherd, J. W. and Davies, G. M. 1980: The deterioration of verbal descriptions of faces over different delay intervals. Journal of Police Science and Administration, 8, 101-6.

Emmorey, K. 2002: Language, Cognition, and the Brain: Insights from Sign Language Research. Mahwah, NJ: Lawrence Erlbaum Associates.

Everett, D. 2005: Cultural constraints on grammar and cognition in Pirahã. Current Anthropology, 46, 621-46.

Evans-Pritchard, E. E. 1934: Imagery in Ngok Dinka cattle-names. Bulletin of the School of Oriental Studies, London Institution, 7, 623-8.

Fagan, J. F. and Singer, L. T. 1979: The role of simple feature differences in infants' recognition of faces. Infant Behavior and Development, 2, 39-45.

Fitch, W. T. and Reby, D. 2001: The descended larynx is not uniquely human. Proceedings of the Royal Society, Biological Sciences, 268, 1669-75.

Fodor, J. 1983: The Modularity of Mind. Cambridge, MA: MIT Press.

Gage, J. 1995: Colour and Culture. London: Thames \& Hudson.

Gauker, C. 1994: Thinking Out Loud: An Essay on the Relation between Thought and Language. Princeton, NJ: Princeton University Press.

Gentaz, E. and Hatwell, W. 2004: Geometrical haptic illusions: the role of exploration in the Müller-Lyter, vertical-horizontal, and Delboeuf illusions. Psychonomic Bulletin E Review, 11, 31-40.

Gescheider, G. A. 1997: Psychophysics: The Fundamentals, 3rd edn. Hillsdale, NJ: Erlbaum.

Gilad, Y. et al. 2004: Loss of olfactory receptor genes coincides with the acquisition of full trichromatic vision in primates. PLoS Biology 2, 0120-5.

Goldin-Meadow, S. 2007: The challenge: some properties of language can be learned without linguistic input. The Linguistic Review, 24, 449-53.

Golland, Y., Bentin, S., Gelbard, H., Benjamini, Y., Heller, R., Nir, Y., Hasson., U. and Malach, R. 2007: Extrinsic and intrinsic systems in the posterior cortex of the human brain revealed during natural sensory stimulation. Cerebral Cortex, 17, 766-77.

Gunther, Y. (ed.) 2003: Essays on Nonconceptual Content. Cambridge, MA: MIT Press.

Haun, D. B. M., Rapold, C., Call, J., Janzen, G. and Levinson, S. C. 2006: Cognitive cladistics and cultural override in Hominid spatial cognition. PNAS, 103, 17568-73.

Haxby, J. V., Hoffman, E. A. and Gobbini, M. I. 2000: The distributed human neural system for face perception. Trends in Cognitive Sciences, 6, 223-33.

Henning, H. 1916: Der Geruch. Leipzig: Barth.

von Hornbostel, E. M. 1927: The unity of the senses. Psyche, 83-9.

Howes, D. 2006: Cross-talk between the senses. The Senses \& Society, 1, 381-90.

Jackendoff, R. 2007: Language, Consciousness, Culture. Cambridge, MA: MIT Press.

Jakab, Z. 2001: Color Experience: Empirical Evidence Against Representational Externalism. $\mathrm{PhD}$ thesis, Carleton University, Ottawa. 
Khan, R. M., Luk, C., Flinker, A., Aggarwal, A, Lapid, H., Haddad, R. and Sobel, N. 2007: Predicting odor pleasantness from odorant structure: pleasantness as a reflection of the physical world. Journal of Neuroscience, 27, 10015-23.

Kukla, A. 2005: Ineffability and Philosophy. London: Routledge.

Laming, D. 1989: Experimental evidence for Fechner's and Stevens's laws. Behavioral and Brain Sciences, 12, 277-81.

Landau, B. and Gleitman, L. 1985: Language and Experience. Cambridge, MA: Harvard University Press.

Landau, B. and Jackendoff, R. 1993: 'What' and 'where' in spatial language and spatial cognition. Behavioral and Brain Sciences, 16, 217-65.

Lantz, D. and Stefflre, V. 1964: Language and cognition revisited. Journal of Abnormal and Social Psychology, 69, 472-81.

Lenneberg, E. H. 1953: Cognition in ethnolinguistics. Language, 29, 463-71.

Lenneberg, E. H. and Roberts, J. M. 1956: The Language of Experience: A Study in Methodology. Bloomington, IN: Indiana University Publications in Anthropology and Linguistics 13.

Levine, S. B. 2005: What is love anyway? Journal of Sex \& Marital Therapy, 31, 143-51.

Levinson, S. C. 2000: Yélî Dnye and the theory of color terms. Journal of Linguistic Anthropology, 10, 1-53.

Levinson, S. C. 2003: Space in Language and Cognition: Explorations in Cognitive Diversity. Cambridge: Cambridge University Press.

Lewkowicz, D. J. 2011: The biological implausibility of the nature-nurture dichotomy and what it means for the study of infancy. Infancy, 16, 331-67.

Lorig, T. S. 1999. On the similarity of odor and language perception. Neuroscience $\mathcal{E}$ Biobehavioral Reviews, 23, 391-98.

Lucy, J. A. 1992: Language Diversity and Thought: A Reformulation of the Linguistic Relativity Hypothesis. Cambridge: Cambridge University Press.

Lucy, J. A. and Shweder, R. A. 1979: Whorf and his critics: linguistic and nonlinguistic influences on color memory. American Anthropologist, 81, 581-615.

Lupyan, G. 2008: From chair to 'chair': a representational shift account of object labeling effects on memory. Journal of Experimental Psychology: General, 137, 348-69.

Majid, A., Bowerman, M., Kita, S., Haun, D. B. M. and Levinson, S. C. 2004: Can language restructure cognition? The case for space. Trends in Cognitive Science, 8, 108-14.

Majid, A. and Levinson, S. C. 2008: Language does provide support for basic tastes. Behavioral and Brain Sciences, 31, 86-7.

Majid, A. and Levinson, S. C. (eds) 2011: The senses in language and culture. The Senses \& Society [Special Issue], 6, 5-18.

Marks, L. E. 1978: The Unity of the Senses: Interrelations Among the Modalities. New York: Academic Press. 
Marks, L. E. 1989: On cross-modal similarity: the perceptual structure of pitch, loudness, and brightness. Journal of Experimental Psychology: Human Perception and Performance, $15,586-602$.

Maurer, D., Gibson, L. C. and Spector, F. 2012: Infant synaesthesia: New insights into the development of multisensory perception. In A. J. Bremner, D. J. Lewkowicz, and C. Spence (eds), Multisensory Development. Oxford: Oxford University Press.

Mithen, S. J. 1996: The Prehistory of the Mind: The Cognitive Origins of Art, Religion and Science. Cambridge: Cambridge University Press.

Moore, T. and Carling, C. 1988: The Limitations of Language. London: Macmillan.

Murphy, V. N. 2011: Olfactory maps in the brain. Annual Review of Neuroscience, 34, $233-58$.

O’Toole, A. J., Roark, D. A. and Abdi, H. 2002: Recognizing moving faces: a psychological and neural synthesis. Trends in Cognitive Sciences, 6, 261-66.

Perl, E. R. 2007: Ideas about pain: a historical view. Nature Reviews Neuroscience, 8, $71-80$.

Regier, T. and Kay, P. 2009: Language, thought, and color: Whorf was half right. Trends in Cognitive Sciences, 13, 439-46.

Roberson, D., Hyensou, P. and Hanley, J. R. 2008: Categorical perception of colour in the left and right visual field is verbally mediated: evidence from Korean. Cognition, $107,752-62$.

Searle, J. 1969: Speech Acts: An Essay in the Philosophy of Language. Cambridge: Cambridge University Press.

Simner, J. 2007: Beyond perception: synaesthesia as a psycholinguistics phenomenon. Trends in Cognitive Sciences, 11, 23-9.

Simner, J., Sagiv, N., Mulvenna, C., Tsakanikos, E., Witherby, S.A., Fraser, C., Scott, K. and Ward, J. 2006: Synaesthesia: the prevalence of atypical cross-modal experiences. Perception, 35, 1024-33.

Simner, J., Harrold, J., Creed, H., Monro, L. and Foulkes, L. 2009: Early detection of markers for synaesthesia in childhood populations. Brain, 132, 57-64.

Spelke, E. and Tsivkin, S. 2001: Initial knowledge and conceptual change. In M. Bowerman and S. C. Levinson (eds), Language Acquisition and Conceptual Development (pp. 70-97). Cambridge: Cambridge University Press.

Spence, C. 2011: Crossmodal Correspondences: a tutorial review. Attention, Perception, \& Psychophysics, 73, 971-95.

Stalnaker, R. 1998: What might conceptual content be? In E. Villanueva (ed.), Concepts. Philosophical Issues, 9. Atascadero, CA: Ridgeview.

Stevens, S. S. 1957: On the psychophysical law. Psychological Review, 64, 530-41.

Stevens, S. S. 1975: Psychophysics. New York: Wiley \& Sons.

Stoddart, D. M. 1990: The Scented Ape: The Biology and Culture of Human Odour. Cambridge: Cambridge University Press. 
Tarski, A. 1956: Logic, Semantics, Metamathematics. Oxford: Clarendon Press.

Taub, S. 2001: Language from the Body: Iconicity and Metaphor in American Sign Language. Cambridge: Cambridge University Press.

Walsh, V. 2003: A theory of magnitude: Common cortical metrics of time, space, and quantity. Trends in Cognitive Sciences, 7, 483-88.

Ward, J., Huckstep, B. and Tsakanikos, E. 2006: Sound-colour synaesthesia: to what extent does it use cross-modal mechanisms common to us all? Cortex, 42, 264-80.

Weber, E. H. 1834: De Tactu. Annotationes Anatomicae et Physiologicae. Leipzig: Koehler.

Woodward, J. 1989: Basic color term lexicalization across Sign Languages. Sign Language Studies, 63, 145-52.

Wolff, P. and Holmes, K. J. 2011: Linguistic relativity. Wiley Interdisciplinary Reviews: Cognitive Science, 2, 253-65.

Yeshurun, Y. and Sobel, N.: 2010: An odor is not worth a thousand words: from multidimensional odors to unidimensional odor objects. Annual Review of Psychology, 61, 219-41.

Zélanti, P. S. and Droit-Volet, S. 2012: Auditory and visual differences in time perception? An investigation from a developmental perspective with neuropsychological tests. Journal of Experimental Child Psychology, 112, 296-311. 